\title{
Three-quantifier sentences
}

by

\author{
Harvey M. Friedman (Columbus, OH)
}

\begin{abstract}
We give a complete proof that all 3-quantifier sentences in the primitive notation of set theory $(\epsilon,=)$, are decided in ZFC, and in fact in a weak fragment of ZF without the power set axiom. We obtain information concerning witnesses of 2-quantifier formulas with one free variable. There is a 5 -quantifier sentence that is not decided in ZFC (see [2]).
\end{abstract}

1. Preliminaries. [4] presents an argument that all 3-quantifier sentences in the primitive notation of set theory $(\epsilon,=)$ are decided in ZFC. In the author's words, not all of the details are presented:

"It is tedious but involves no difficulty to verify that if. .." (p. 5, line 14).

"This can be verified by considering all the possible cases, but is quite clear if considered carefully. So we omit what would be a very long verification" (p. 8, end).

We give a complete proof that all 3 -quantifier sentences in set theory based on $\epsilon,=$ are decided in ZFC. In fact, we show that all sentences of somewhat higher complexity are decided in a weak fragment $T$ of ZF without the power set axiom. We also give some strong information about witnessing 2 -quantifier formulas. Our main results are summarized in Theorem 66. We do not use ideas from [4].

We work entirely within the primitive language of set theory, which is standard predicate calculus with equality and $\in$, using the standard quantifiers $\forall, \exists$, and the standard connectives $\neg, \wedge, \vee, \rightarrow, \leftrightarrow$. We assume the usual complete axioms and rules of classical logic.

We let $T$ be the following weak set theory:

1. Extensionality.

2. Pairing.

3. Union.

4. Infinity (modified).

2000 Mathematics Subject Classification: Primary 03E30; Secondary 03B25.

This research was partially supported by NSF Grant DMS-9970459. 
5. Foundation.

6. Bounded Separation.

Pairing asserts the existence of $\{a, b\}$. Union asserts the existence of $\cup a$.

The usual formulation of Infinity in ZF is the existence of a set containing $\emptyset$ and closed under the operation that sends $x$ to $x \cup\{x\}$. Because we are not using Replacement, we use the stronger version of infinity that asserts the existence of a least set containing $\emptyset$ and closed under the operation that sends $x, y$ to $x \cup\{y\}$.

Foundation asserts that every nonempty set has an epsilon minimal element.

Bounded Separation asserts the existence of $\{x \in a: \varphi\}$, where $\varphi$ is a formula in which all quantifiers are bounded, i.e., of the forms $(\forall u \in v)$, $(\exists u \in v)$, where $u, v$ are distinct variables.

In $T$, we can prove the existence of a least nonempty transitive set closed under power set, which we write as $V(\omega)$. In $T$, we can develop all of the basic facts about $V(\omega)$ and its elements, as well as define subsets of $V(\omega)$ and functions on $V(\omega)$ by recursion. Since this derivation is not completely straightforward, we give a sketch of the steps used.

a. Let $A$ be given by Infinity. Thus $\emptyset \in A,(\forall x, y \in A)(x \cup\{y\} \in A)$ and for all $B$ satisfying these conditions, we have $A \subseteq B$.

b. Show that $A$ is transitive. Show that $(\forall x, y \in A)(\{x, y\} \in A \wedge x \cup y$ $\in A \wedge x \backslash y \in A)$.

c. Define $\omega=\{x \in A: x$ is epsilon connected and transitive $\}$. Show that $\emptyset \in \omega,(\forall x \in \omega)(x \cup\{x\} \in \omega), \omega$ is epsilon connected and transitive. Show that $(\forall x \in \omega)(\exists y)(x=\emptyset \vee x=y \cup\{y\})$.

d. Show that if $f$ is a partial function from an element of $\omega$ into $A$, then $f$ and the range of $f$ are elements of $A$. Show that every element of $A$ is the range of a bijection from an element of $\omega$, where the bijection is an element of $A$.

e. Show that every subset of every element of $A$ is an element of $A$. Show that the power set of every element of $A$ exists. Show that for all $x, y \in A$, if the power set of $x$ is an element of $A$, then the power set of $x \cup\{y\}$ is an element of $A$. Show that the power set of every element of $A$ is an element of $A$.

f. Show that there is a unique function $f: \omega \rightarrow A$ such that $f(\emptyset)=\emptyset$ and $f(x \cup\{x\})=$ the power set of $f(x)$.

g. Show that the union of the range of $f$ is $A$. Show that $A$ is the least nonempty transitive set which is closed under power set. Write $A=V(\omega)$.

$\mathrm{h}$. Show the following. Let $\varphi(x, y)$ be a bounded formula with parameters such that $(\forall x \in V(\omega))(\exists ! y \in V(\omega))(\varphi(x, y))$. There exists a unique $f$ : $V(\omega) \rightarrow V(\omega)$ such that $f(x)=$ the unique $y \in V(\omega)$ with $\varphi(x, f \mid x)$.

Alternatively, we can use the following fragment of ZF without power set, with the standard axiom of infinity: 
1. Extensionality.

2. Pairing.

3. Union.

4. Infinity (standard).

5. Foundation.

6. Full Separation.

7. $\Sigma_{1}$ Replacement.

Here $\Sigma_{1}$ Replacement takes the form

$$
(\forall x \in y)(\exists ! z)(\varphi) \rightarrow(\exists w)(\forall x \in y)(\exists z \in w)(\varphi),
$$

where $\varphi$ is a bounded formula in which $w$ is not free. This latter system logically implies $T$.

Since the focus of the paper is on the decidability of 3-quantifier sentences, we strictly follow the simplifying convention that all formulas will use at most the three distinct variables $x, y, z$. Letters such as $u, v, w$ are used as metavariables over the official variables $x, y, z$, or variables used to carry out proof sketches that are to take place in $T$.

The previous paragraph does not apply to the proofs within $T$. Any formulas in the language of $T$ are allowed to be present in proofs within $T$.

The basic formulas are the atomic formulas that use at most $x, y, z$, together with their negations.

The $\Delta_{0}$ formulas are the quantifier free formulas that use at most $x, y, z$.

We use the notation $\exists, \forall, \exists \exists, \exists \forall, \forall \exists, \forall \forall, \exists \exists \exists, \exists \exists \forall, \exists \forall \exists, \exists \forall \forall$, etc., to denote the obvious prenex classes of formulas that use at most $x, y, z$, where the quantifiers, from left to right, read $z, y z$, or $x y z$. We will follow the convention that there cannot be any missing quantifiers.

We also consider some thickened complexity classes. For example, we write $\forall \exists \wedge \ldots \wedge \forall \exists$ for the class of all conjunctions of $\forall \exists$ formulas. We also write $\exists(\forall \exists \wedge \ldots \wedge \forall \exists)$ for the class of all single existentially quantified formulas over $\forall \exists \wedge \ldots \wedge \forall \exists$. Note that the former have at most the free variable $x$, and the latter are sentences.

We will prove that all sentences in $\exists(\forall \exists \wedge \ldots \wedge \forall \exists), \exists(\exists \exists \wedge \ldots \wedge \exists \exists)$ are decided in $T$.

We use $u$ inc $w$ for " $u, w$ are incomparable", i.e., $u \notin w \wedge w \notin u \wedge u \neq w$. We use $u \operatorname{comp} w$ for " $u, w$ are comparable", i.e., $u \in w \vee w \in u \vee u=w$. Here $u, w$ are metavariables over the variables $x, y, z$.

The complete conjunctions are the conjunctions of basic formulas, $R$, where

(i) all conjuncts of $R$ use two distinct variables;

(ii) the $x y$ part of $R$ is exactly one among $x \in y, y \in x, x=y, x$ inc $y$; 
(iii) the $x z$ part of $R$ is exactly one among $x \in z, z \in x, x=z, x$ inc $z$;

(iv) the $y z$ part of $R$ is exactly one among $y \in z, z \in y, y=z, y$ inc $z$.

Example: $x \in y \wedge x=z \wedge y$ inc $z$ is a complete conjunction, which is an abbreviation for $x \in y \wedge x=z \wedge y \notin z \wedge z \notin y \wedge y \neq z$. Note that $x \in y \wedge x=z \wedge y$ inc $z$ is refutable.

Throughout the paper, all conjunctions and all disjunctions are nonempty. This means that we have to take some care about degenerate cases.

A full conjunction (of basic formulas) is a conjunction that includes exactly one element from each $\{\varphi, \neg \varphi\}$, where $\varphi$ is atomic. (As always, we only allow the variables $x, y, z$.)

Note the distinction between complete conjunctions and full conjunctions. We say that a formula $\varphi$ is decided in $T$ if $T$ proves $\varphi$ or $T$ proves $\neg \varphi$.

We say that $\varphi$ is refuted in $T$ if $T$ proves $\neg \varphi$.

We say that two formulas $\varphi, \psi$ are equivalent in $T$ if $T$ proves $\varphi \leftrightarrow \psi$.

We say that $\varphi$ implies $\psi$ in $T$ if $T$ proves $\varphi \rightarrow \psi$. Note that the notions of "decided" and "equivalent" are particularly strong when applied to formulas with free variables, as there are implicit universal quantifiers.

Lemma 1. Every $\Delta_{0}$ formula is equivalent, in $T$, to a disjunction of complete conjunctions.

Proof. We first show that every full conjunction $\varphi$ is equivalent to a complete conjunction. If $\varphi$ is refutable then we are done by taking the complete conjunction $x=y \wedge y=z \wedge x \in z$. We can assume that $\varphi$ is not refutable, and so $\varphi$ has each $u=u, u \notin u$. We eliminate them from $\varphi$, maintaining equivalence in $T$. Now look at the $x y$ part of $\varphi$. If it has no positive term then the $x y$ part of $\varphi$ is equivalent to $x$ inc $y$. If it has $x \in y$, then it has no other positive terms, and hence is equivalent to $x \in y$. If it has $y \in x$, then it has no other positive terms, and is equivalent to $y \in x$. It has $x=y$ if and only if it has $y=x$, in which case it has no other positive terms, and is equivalent to $x=y$. Argue the same way for the $x z$ part and the $y z$ part.

By disjunctive normal form theory, the $\Delta_{0}$ formula is either the negation of a tautology or tautologically equivalent to a disjunction of full conjunctions - and hence equivalent, in $T$, to a disjunction of complete conjunctions. Since there is a complete conjunction that is equivalent, in $T$, to the negation of a tautology, the proof is complete.

2. One existential quantifier. We refer the reader to the A list of formulas in the Appendix.

Lemma 2. Let $\alpha$ be from $x \in z, z \in x, x=z, x$ inc $z$, and $\beta$ be from $y \in z, z \in y, y=z, y$ inc $z$. Then $(\exists z)(\alpha \wedge \beta)$ is equivalent, in $T$, to a formula on the A list. 
Proof. We argue by cases according to $\alpha$.

Case 1: $x \in z$. With $y \in z$ we get truth. With $z \in y$ we get $x \in \cup y$. With $y=z$ we get $x \in y$. With $y$ inc $z$ we get $x \neq y$ by setting $z=\{x,\{y\}\}$.

Case 2: $z \in x$. With $y \in z$ we get $y \in \cup x$. With $z \in y$ we get $x \cap y \neq \emptyset$. With $y=z$ we get $y \in x$. With $y$ inc $z$ we get $(\exists z \in x)(z$ inc $y)$.

Case 3: $x=z$. With $y \in z$ we get $y \in x$. With $z \in y$ we get $x \in y$. With $y=z$ we get $x=y$. With $y$ inc $z$ we get $x$ inc $y$.

Case 4: $x$ inc $z$. With $y \in z$ we get $x \neq y$ by setting $z=\{y,\{x\}\}$. With $z \in y$ we get $(\exists z \in y)(z$ inc $x)$. With $y=z$ we get $x$ inc $y$. With $y$ inc $z$ we get truth by setting $z=\{\{x, y\}\}$.

Lemma 3. Let $R$ be a complete conjunction. Then $(\exists z)(R(x, y, z))$ is equivalent, in $T$, to a formula of the form $x \in y \wedge \varphi, y \in x \wedge \varphi, x=y \wedge \varphi$, or $x$ inc $y \wedge \varphi$, where $\varphi$ is a formula on the $\mathrm{A}$ list.

Proof. By predicate calculus manipulations, we can pull out the $x y$ part of $R$, which is $x=y, x \in y, y \in x$, or $x$ inc $y$. The remaining part of $R$ consists of the $x z$ part and the $y z$ part. Now apply Lemma 2 .

Lemma 4. Every $\exists$ formula is equivalent, in $T$, to a formula

$$
\left(x \in y \rightarrow \varphi_{1}\right) \wedge\left(y \in x \rightarrow \varphi_{2}\right) \wedge\left(x=y \rightarrow \varphi_{3}\right) \wedge\left(x \text { inc } y \rightarrow \varphi_{4}\right),
$$

where each $\varphi$ is a disjunction of formulas on the A list. Every $\forall \exists$ formula is equivalent, in $T$, to a formula

$$
\begin{aligned}
(\forall y)\left(x \in y \rightarrow \varphi_{1}\right) \wedge(\forall y)(y & \left.\in x \rightarrow \varphi_{2}\right) \\
& \wedge(\forall y)\left(x=y \rightarrow \varphi_{3}\right) \wedge(\forall y)\left(x \text { inc } y \rightarrow \varphi_{4}\right),
\end{aligned}
$$

where each $\varphi$ is a disjunction of formulas on the $\mathrm{A}$ list.

Proof. We remind the reader that the existentially quantified formula in an $\exists$ formula must be $z$. The second claim follows immediately from the first. Let $(\exists z)(\gamma)$ be given, where $\gamma$ is $\Delta_{0}$. Find a disjunction of complete conjunctions, equivalent to $\gamma$, according to Lemma 1. Then $(\exists z)(\gamma)$ is equivalent to a disjunction $(\exists z)\left(\psi_{1}\right) \vee \ldots \vee(\exists z)\left(\psi_{n}\right)$, where the $\psi$ 's are complete conjunctions. By Lemma 3, this is equivalent to a disjunction of formulas of the four forms appearing in Lemma 3, where each $\varphi$ is on the A list. By distributivity, we can put this in the form $\left(x \in y \wedge \varphi_{1}\right) \vee\left(y \in x \wedge \varphi_{2}\right) \vee\left(x=y \wedge \varphi_{3}\right) \vee$ $\left(x\right.$ inc $\left.y \wedge \varphi_{4}\right)$, where each $\varphi$ is a disjunction of formulas on the $\mathrm{A}$ list. This is obviously equivalent to $\left(x \in y \rightarrow \varphi_{1}\right) \wedge\left(y \in x \rightarrow \varphi_{2}\right) \wedge\left(x=y \rightarrow \varphi_{3}\right) \wedge$ $\left(x\right.$ inc $y \rightarrow \varphi_{4}$ ), since $x \in y, y \in x, x=y, x$ inc $y$ are exhaustive and mutually exclusive.

In light of Lemma 4, we will make four analyses, one for each of the four clauses. These analyses are presented in Sections 3-6. 
For these analyses, it is convenient to move to the dual, and work with $(\exists y)(x \in y \wedge \varphi),(\exists y)(y \in x \wedge \varphi),(\exists y)(x=y \wedge \varphi),(\exists y)(x$ inc $y \wedge \varphi)$, where $\varphi$ is a conjunction of formulas from the $\mathrm{A}^{*}$ list in the Appendix.

Lemma 5. Every $\exists \forall$ formula is equivalent, in $T$, to a formula $(\exists y)\left(x \in y \wedge \varphi_{1}\right) \vee(\exists y)\left(y \in x \wedge \varphi_{2}\right) \vee(\exists y)\left(x=y \wedge \varphi_{3}\right) \vee(\exists y)\left(x\right.$ inc $\left.y \wedge \varphi_{4}\right)$, where each $\varphi$ is a conjunction of formulas from the $\mathrm{A}^{*}$ list. Every such disjunction is equivalent, in $T$, to a $\exists \forall$ formula.

Proof. By Lemma 4 and duality, each $\exists \forall$ formula can be put in this form, provided that we use the list of negations of formulas on the $\mathrm{A}$ list. The $\mathrm{A}^{*}$ list is precisely the list of negations of formulas on the A list, but with $x \neq x$ missing. But $x \neq x$ is not needed since it is equivalent, in $T$, to a conjunction of formulas from the $\mathrm{A}^{*}$ list.

The disjunction appearing in the lemma is obviously equivalent to

$$
(\exists y)\left(\left(x \in y \rightarrow \varphi_{1}\right) \wedge\left(y \in x \rightarrow \varphi_{2}\right) \wedge\left(x=y \rightarrow \varphi_{3}\right) \vee\left(x \text { inc } y \rightarrow \varphi_{4}\right)\right) .
$$

Since each $\varphi$ is $\forall$, we see that the implications are $\forall$, and hence the conjunction is also $\forall$.

The next four sections treat each of the four disjuncts appearing in Lemma 5 .

3. $(\exists \boldsymbol{y})(\boldsymbol{x} \in \boldsymbol{y} \wedge \boldsymbol{\varphi})$. Here $\varphi$ is a conjunction of an $\mathrm{A}^{*}$ sublist, i.e., of a sublist of the $\mathrm{A}^{*}$ list. We say that (the conjunction of) an $\mathrm{A}^{*}$ sublist, $\varphi$, yields $\psi$ if $(\exists y)(x \in y \wedge \varphi)$ is equivalent, in $T$, to $\psi$.

We wish to compile the various $\psi$ that are yielded by the various $\mathrm{A}^{*}$ sublists $\varphi$. We find convenient representatives up to equivalence of the yielded $\psi$.

Clearly truth and falsity (e.g., $x=x, x \neq x$ ) are among the $\psi$ that are yielded. We take these for granted. In light of this convention, we do not need to work with those formulas from the $\mathrm{A}^{*}$ list which are refuted in $T$ by $x \in y$. Also we do not need to work with those formulas from the $\mathrm{A}^{*}$ list which are provable in $T$ from $x \in y$ since $(\exists y)(x \in y)$ is provable in $T$. Therefore in this section we work within the following $\mathrm{A}^{*}$ sublist:

$$
\begin{aligned}
& x \cap y=\emptyset \\
& x \notin \cup y \\
& (\forall z \in x)(z \operatorname{comp} y) \\
& (\forall z \in y)(z \operatorname{comp} x) . \\
& \text { LEMma 6. } x \cap y=\emptyset, x \notin \cup y,(\forall z \in y)(z \text { comp } x) \text { yields truth. } \\
& \text { Proof. Set } y=\{x\} \text {. - } \\
& \text { LeMma 7. } x \notin \cup y,(\forall z \in y)(z \text { comp } x),(\forall z \in x)(z \text { comp y) yields truth. } \\
& \text { Proof. Set } y=x \cup\{x\} . \text { - }
\end{aligned}
$$


Lemma 8. $x \cap y=\emptyset,(\forall z \in x)(z$ comp $y)$ yields $x=\emptyset$. Also $x \cap y=\emptyset$, $x \notin \cup y,(\forall z \in x)(z$ comp $y),(\forall z \in y)(z$ comp $x)$ yields $x=\emptyset$.

Proof. Let $z \in x$. Since $x \cap y=\emptyset$, we have $z \notin y$. Also since $x \in y$, we see that $z=y$ and $y \in z$ are impossible. Hence $x=\emptyset$. For the converse, set $y=\{\emptyset\}$.

Here is the yield table.

1. $x \cap y=\emptyset$. Yields truth.

2. $x \notin \cup y$. Yields truth.

3. $(\forall z \in x)(z$ comp $y)$. Yields truth.

4. $(\forall z \in y)(z \operatorname{comp} x)$. Yields truth.

5. $x \cap y=\emptyset, x \notin \cup y$. Yields truth.

6. $x \cap y=\emptyset,(\forall z \in x)(z \operatorname{comp} y)$. Yields $x=\emptyset$.

7. $x \cap y=\emptyset,(\forall z \in y)(z \operatorname{comp} x)$. Yields truth.

8. $x \notin \cup y,(\forall z \in x)(z$ comp $y)$. Yields truth.

9. $x \notin \cup y,(\forall z \in y)(z \operatorname{comp} x)$. Yields truth.

10. $(\forall z \in x)(z \operatorname{comp} y),(\forall z \in y)(z \operatorname{comp} x)$. Yields truth.

11. $x \cap y=\emptyset, x \notin \cup y,(\forall z \in x)(z$ comp $y)$. Yields $x=\emptyset$.

12. $x \cap y=\emptyset, x \notin \cup y,(\forall z \in y)(z \operatorname{comp} x)$. Yields truth.

13. $x \cap y=\emptyset,(\forall z \in x)(z$ comp $y),(\forall z \in y)(z \operatorname{comp} x)$. Yields $x=\emptyset$.

14. $x \notin \cup y,(\forall z \in x)(z$ comp $y),(\forall z \in y)(z$ comp $x)$. Yields truth.

15. $x \cap y=\emptyset, x \notin \cup y,(\forall z \in x)(z$ comp $y),(\forall z \in y)(z$ comp $x)$. Yields $x=\emptyset$.

4. $(\exists \boldsymbol{y})(\boldsymbol{y} \in \boldsymbol{x} \wedge \boldsymbol{\varphi})$. Recall the terminology and conventions of Section 3 . We work within the following $\mathrm{A}^{*}$ sublist:

$$
\begin{aligned}
& x \cap y=\emptyset \\
& y \notin \cup x \\
& x \text { comp y } \\
& (\forall z \in x)(z \text { comp } y) \\
& (\forall z \in y)(z \text { comp } x) .
\end{aligned}
$$

We need to work with $x$ comp $y$, even though it is provable, in $T$, from $y \in x$, since $(\exists y)(y \in x)$ is not itself provable in $T$.

LEMMA 9. In the presence of $y \in x,(\forall z \in y)(z$ comp $x)$ is equivalent, in $T$, to $y \subseteq x$.

Proof. Let $z \in y$. Then $z=x$ and $x \in z$ are each impossible. So $z \in x$. Hence $y \subseteq x$.

In light of Lemma 9, we work with the following $\mathrm{A}^{*}$ sublist:

$$
\begin{aligned}
& x \cap y=\emptyset \\
& y \notin \cup x
\end{aligned}
$$




$$
\begin{aligned}
& y \subseteq x \\
& x \text { comp } y \\
& (\forall z \in x)(z \operatorname{comp} y) .
\end{aligned}
$$

It is clear that $(\exists y)(y \in x \wedge x \operatorname{comp} y \wedge \varphi)$ is equivalent, in $T$, to the formula $(\exists y)(y \in x \wedge \varphi)$. Therefore, we have only to work with $x$ comp $y$ once. In fact, we merely have to note that $x \operatorname{comp} y$ yields $x \neq \emptyset$, and then we can remove $x$ comp $y$ from consideration. In light of Lemma 10 below, we can ignore $x$ comp $y$ entirely. Thus we work only with the $\mathrm{A}^{*}$ sublist

$$
\begin{aligned}
& x \cap y=\emptyset \\
& y \notin \cup x \\
& y \subseteq x \\
& (\forall z \in x)(z \text { comp } y) .
\end{aligned}
$$

In many of these cases, we just make an obvious restatement of the formula $(\exists y)(y \in x \wedge \varphi)$ in English. The cases where we can do better are presented as lemmas.

Lemma 10. $x \cap y=\emptyset$ yields $x \neq \emptyset$.

Proof. Since $y \in x$, we have $x \neq \emptyset$. For the converse, set $y$ to be a minimal element of $x$.

Lemma 11. $x \cap y=\emptyset,(\forall z \in x)(z$ comp $y)$ yields "some element of $x$ lies in all other elements of $x "$.

Proof. From the list, we see that $y$ is a minimal element of $x$ that is comparable with all elements of $x$. Since $y$ is minimal, no element of $x$ lies in $y$. Hence $y$ lies in all other elements of $x$. Conversely, let $y \in x$ lie in all other elements of $x$. Then $y$ is epsilon minimal.

Lemma 12. $x \cap y=\emptyset, y \subseteq x$ yields $\emptyset \in x$.

Proof. From the list, we see that $y=\emptyset$. Hence $\emptyset \in x$. For the converse, set $y=\emptyset$.

Lemma 13. $y \notin \cup x,(\forall z \in x)(z$ comp $y)$ yields " $x$ has an epsilon maximum element".

Proof. Let $z \in x$. Then $z=y$ or $z \in y$. So $y$ is an (the) epsilon maximum element. For the converse, let $y$ be the epsilon maximum element.

Lemma 14. $x \cap y=\emptyset, y \notin \cup x,(\forall z \in x)(z$ comp $y)$ yields " $x$ is a singleton".

Proof. As in Lemma 13, $y$ must be an epsilon maximal element of $x$. By $x \cap y=\emptyset$, we see that $x=\{y\}$.

Lemma 15. $x \cap y=\emptyset, y \notin \cup x, y \subseteq x$ yields " $\emptyset$ is an epsilon maximal element of $x "$. 
Proof. From the list, we see that $y=\emptyset$, and so $\emptyset \in x$. Also $\emptyset$ is not in $\cup x$. For the converse, set $y=\emptyset$.

Lemma 16. $x \cap y=\emptyset, y \notin \cup x, y \subseteq x,(\forall z \in x)(z$ comp $y)$ yields $x=\{\emptyset\}$.

Proof. From the list we obtain $y=\emptyset$. From Lemma 14, we have $x=\{y\}$. For the converse, set $y=\emptyset$.

LEMma 17. $x \cap y=\emptyset, y \notin \cup x$ yields "some epsilon minimal element of $x$ is an epsilon maximal element of $x "$.

Proof. By $x \cap y=\emptyset, y$ is an epsilon minimal element of $x$. By $y \notin \cup x$, $y$ is an epsilon maximal element of $x$. The converse is also immediate.

Lemma 18. $x \cap y=\emptyset, y \subseteq x,(\forall z \in x)(z$ comp $y)$ yields $" \emptyset \in x$ is an element of all other elements of $x "$.

Proof. By Lemma 11, we see that $y$ is a minimal element of $x$ that is an element of all other elements of $x$. Since $y \subseteq x$, we have $y=\emptyset$. Conversely, set $y=\emptyset$.

Lemma 19. $y \notin \cup x, y \subseteq x,(\forall z \in x)(z$ comp $y)$ yields " $x$ is of the form $b \cup\{b\} "$.

Proof. By Lemma 13, we see that $y$ is the maximum element of $x$, i.e., $x \subseteq y \cup\{y\}$ and $y \in x$. By $y \subseteq x$, we have $x=y \cup\{y\}$.

Here is the yield table.

1. $x \cap y=\emptyset$. Yields $x \neq \emptyset$.

2. $y \notin \cup x$. Yields " $x$ has an epsilon maximal element".

3. $y \subseteq x$. Yields "some subset of $x$ lies in $x$ ".

4. $(\forall z \in x)(z \operatorname{comp} y)$. Yields "some element of $x$ is comparable with all elements of $x "$.

5. $x \cap y=\emptyset, y \notin \cup x$. Yields "some epsilon minimal element of $x$ is an epsilon maximal element of $x$ ".

6. $x \cap y=\emptyset, y \subseteq x$. Yields $\emptyset \in x$.

7. $x \cap y=\emptyset,(\forall z \in x)(z$ comp $y)$. Yields "some element of $x$ lies in all other elements of $x$ ".

8. $y \notin \cup x, y \subseteq x$. Yields "some subset of $x$ is an epsilon maximal element of $x$ ".

9. $y \notin \cup x,(\forall z \in x)(z$ comp $y)$. Yields " $x$ has an epsilon maximum element".

10. $y \subseteq x,(\forall z \in x)(z \operatorname{comp} y)$. Yields "there is a subset of $x$ lying in $x$ which is comparable with every element of $x$ ".

11. $x \cap y=\emptyset, y \notin \cup x,(\forall z \in x)(z$ comp $y)$. Yields " $x$ is a singleton".

12. $x \cap y=\emptyset, y \notin \cup x, y \subseteq x$. Yields " $\emptyset$ is an epsilon maximal element of $x "$. 
13. $x \cap y=\emptyset, y \subseteq x,(\forall z \in x)(z \operatorname{comp} y)$. Yields " $\emptyset \in x$ is an element of all other elements of $x$ ".

14. $y \notin \cup x, y \subseteq x,(\forall z \in x)(z$ comp $y)$. Yields " $x$ is of the form $b \cup\{b\}$ ".

15. $x \cap y=\emptyset, y \notin \cup x, y \subseteq x,(\forall z \in x)(z \operatorname{comp} y)$. Yields $x=\{\emptyset\}$.

In 4, 10, comparable means comp.

5. $(\exists \boldsymbol{y})(\boldsymbol{x}=\boldsymbol{y} \wedge \boldsymbol{\varphi}) .(\exists y)(x=y \wedge \varphi)$ is logically equivalent to $\varphi[y / x]$. So we need only consider the $\varphi[y / x]$, where $\varphi$ is a conjunction of formulas from the $\mathrm{A}^{*}$ list. We first determine those $\varphi[y / x]$ with $\varphi$ on the $\mathrm{A}^{*}$ list that are not decided in $T$. By inspection, this can only be $x=\emptyset$. So $x=\emptyset$ is the only nontrivial yield, the others being truth and falsity.

6. $(\exists \boldsymbol{y})(\boldsymbol{x}$ inc $\boldsymbol{y} \wedge \boldsymbol{\varphi})$. Recall the terminology and conventions of Section 3 . We work with the following $\mathrm{A}^{*}$ sublist:

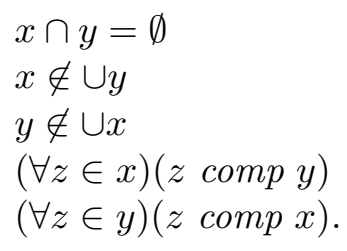

Lemma 20. $x \cap y=\emptyset, x \notin \cup y, y \notin \cup x$ yields truth.

Proof. Set $y=\{\{\{x\}\}\}$.

Lemma 21. $x \notin \cup y, y \notin \cup x,(\forall z \in x)(z$ comp y) yields truth.

Proof. Set $y=x \cup\{\{\{x\}\}\}$.

Lemma 22. $x \cap y=\emptyset, y \notin \cup x,(\forall z \in y)(z$ comp $x)$ yields truth.

Proof. Set $y=\{\{x\}\}$.

Lemma 23. $x \cap y=\emptyset,(\forall z \in x)(z$ comp $y)$ yields "all elements of $x$ have a common element". Same if $x \notin \cup y$ is appended.

Proof. Let $z \in x$. Now $z=y, z \in y$ are impossible. Hence $y \in z$. So $y$ lies in every element of $x$. For the converse, first suppose that $x=\emptyset$. Set $y=\{\{\{\emptyset\}\}\}$. Now suppose that $x \neq \emptyset$. Let $y$ lie in every element of $x$. Hence $x \cap y=\emptyset$. Also, $y \notin x$ since $y$ lies in any element of $x$. In addition, $x \in y$, $x=y, x \in \cup y$ are impossible since $y$ lies in an element of $x$. Therefore $x$ inc $y$.

Lemma 24. The following is provable in T. Let $|x| \geq 2$. Then some proper subset of $x$ does not lie in $x$ and does not lie in $\cup x$.

Proof. There is a conceptually straightforward proof, which, unfortunately, is not carried out in $T$. First suppose that $x$ has an element $u$ of highest rank. Then by rank considerations, $\{u\} \notin x, \cup x$. Now suppose that 
$x$ has no element of highest rank. Let $u \in x$. Then $\operatorname{rk}(x \backslash\{u\})=\operatorname{rk}(x)$, and so again by rank considerations, $x \backslash\{u\} \notin x, \cup x$.

We now present a proof formalizable within $T$. Let $|x| \geq 2$. We first show that $x$ has an element $y$ such that $y$ and $\cup y$ are disjoint from $x$.

Let $A$ be the set of all $z$ such that $z \in x$ or $(z \in \cup x$ and $z$ meets $x)$. Let $y$ be an epsilon minimal element of $A$. By the epsilon minimality of $A$, $y$ is disjoint from $x$.

Case 1: $y \in x$. Suppose $b \in c \in y, b \in x$. Then $c \in \cup x$ and $c$ meets $x$. This contradicts the epsilon minimality of $y$. Thus we have shown that $y$ is disjoint from $x$ and $\cup y$ is disjoint from $x$.

Case 2: $y \notin x$. Since $y \in A$, we see that $y$ meets $x$. This contradicts the epsilon minimality of $y$.

We now fix $y \in x$ such that $y$ and $\cup y$ are disjoint from $x$.

Suppose $x \backslash\{y\} \in x$. Then $x \backslash\{y\}=y$. Since $|x| \geq 2, x$ meets $y$. So $x \backslash\{y\} \notin x$.

Suppose $x \backslash\{y\}$ in $\cup x$. Let $x \backslash\{y\} \in z \in x$. Then $z=y$ and $x \backslash\{y\} \in y$. Since $|x| \geq 2, \cup x$ meets $y$. So $x \backslash\{y\} \notin \cup x$.

LEMMA 25. $x \notin \cup y,(\forall z \in y)(z \operatorname{comp} x)$ yields $x \neq \emptyset,\{\emptyset\}$.

Proof. Let $z \in y$. Then $z=x$ contradicts $x$ inc $y$, and $x \in z$ contradicts $x \notin \cup y$. So $z \in x$. Hence $y \subseteq x$. Hence $y$ must be a proper subset of $x$ that does not lie in $x$. Hence $x \neq \emptyset,\{\emptyset\}$.

For the converse, first assume $|x|=1$ and set $y=\emptyset$. Since $x=\{u\}$, $u \neq \emptyset$, we have $x$ inc $y$.

Now assume $|x| \geq 2$. By Lemma 24, set $y$ to be any proper subset of $x$.

LEMMA 26. $x \cap y=\emptyset, y \notin \cup x,(\forall z \in y)(z$ comp $x)$ yields truth.

Proof. Set $y=\{\{x\}\}$.

LEMMA 27. $y \notin \cup x,(\forall z \in x)(z \operatorname{comp} y),(\forall z \in y)(z$ comp $x)$ yields truth.

Proof. Set $y=x \cup\{\{x\}\}$.

LEMMA 28. $x \cap y=\emptyset, x \notin \cup y,(\forall z \in y)(z \operatorname{comp} x)$ yields $x \neq \emptyset \wedge \emptyset \notin x$.

Proof. By the proof of Lemma 25, $y$ must be a proper subset of $x$ that does not lie in $x$. Hence $y=\emptyset$. Since $x$ inc $y$, we have $x \neq \emptyset$ and $\emptyset \notin x$. For the converse, set $y=\emptyset$.

LEMma 29. $x \cap y=\emptyset, y \notin \cup x,(\forall z \in x)(z$ comp $y)$ yields $x=\emptyset$. Same with $x \notin \cup y$.

Proof. Let $z \in x$. Now $z=y$ is impossible by $x$ inc $y, z \in y$ is impossible by $x \cap y=\emptyset$, and $y \in z$ is impossible by $y \notin \cup x$. So $x=\emptyset$. For the converse, set $y=\{\{\{\emptyset\}\}\}$. 
Lemma 30. $x \cap y=\emptyset,(\forall z \in x)(z$ comp $y),(\forall z \in y)(z$ comp $x)$ yields $x=\emptyset$. Same with $y \notin \cup x$.

Proof. As in the proof of Lemma 23, we find that $y$ lies in every element of $x$. Let $z \in y$. Now $z=x$ and $z \in x$ are each impossible. Therefore $x \in z$. If $x \neq \emptyset$ then write $x \in z \in y \in w \in x$, a contradiction. Therefore $x=\emptyset$. Now suppose $x=\emptyset$. Set $y=\{\{\emptyset\}\}$.

Lemma 31. $x \notin \cup y, y \notin \cup x,(\forall z \in y)(z$ comp $x)$ yields $x \neq \emptyset,\{\emptyset\},\{\{\emptyset\}\}$.

Proof. By the proof of Lemma 25, $x \neq \emptyset,\{\emptyset\}$, and $y$ is a proper subset of $x$ not in $x$. Also $x \neq\{\{\emptyset\}\}$, because otherwise $y=\emptyset$ and $y \in \cup x$. Now suppose $x \neq \emptyset,\{\emptyset\},\{\{\emptyset\}\}$. If $|x|=1$ then take $y=\emptyset$. If $|x| \geq 2$ then by Lemma 24 , take $y$ to be a proper subset of $x$ not lying in $x$ and not lying in $\cup x$.

LEMMA 32. $x \notin \cup y,(\forall z \in x)(z \operatorname{comp} y),(\forall z \in y)(z$ comp $x)$ yields "there is a proper subset $y$ of $x$ not lying in $x$, which is an element of every element of $x \backslash y$ ".

Proof. By the proof of Lemma 25, we deduce that $y$ is a proper subset of $x$ not lying in $x$. Let $z \in x \backslash y$. Now $z \in y, z=y$ are impossible. Hence $y \in z$. For the converse, let $y$ be a proper subset of $x$ not lying in $x$, which is an element of every element of $x \backslash y$. We must verify $(\forall z \in x)(z$ comp $y)$. Let $z \in x$. If $z \notin y$ then $y \in z$.

Lemma 33. $x \cap y=\emptyset, x \notin \cup y, y \notin \cup x,(\forall z \in y)(z$ comp $x)$ yields $x \neq \emptyset$, $\emptyset \notin x, \emptyset \notin \cup x$.

Proof. By Lemma 28, we get $x \neq \emptyset, \emptyset \notin x, y=\emptyset$. Hence $\emptyset \notin \cup x$. Conversely, set $y=\emptyset$.

LEMma 34. $x \cap y=\emptyset, x \notin \cup y,(\forall z \in x)(z$ comp $y),(\forall z \in y)(z \operatorname{comp} x)$ yields falsity.

Proof. By Lemma 30, we get $x=\emptyset$. Let $z \in y$. Now $z=x, z \in x$ are impossible. Hence $x \in z$, contradiction.

Lemma 35. $x \notin \cup y, y \notin \cup x,(\forall z \in x)(z \operatorname{comp} y),(\forall z \in y)(z \operatorname{comp} x)$ yields falsity.

Proof. By Lemma 32, $y$ is a proper subset of $x$ not lying in $x$, which is an element of every element of $x \backslash y$. In particular, $y$ is an element of some element of $x$, violating $y \notin \cup x$.

Here is the yield table.

1. $x \cap y=\emptyset$. Yields truth.

2. $x \notin \cup y$. Yields truth.

3. $y \notin \cup x$. Yields truth. 
4. $(\forall z \in x)(z \operatorname{comp} y)$. Yields truth.

5. $(\forall z \in y)(z \operatorname{comp} x)$. Yields truth.

6. $x \cap y=\emptyset, x \notin \cup y$. Yields truth.

7. $x \cap y=\emptyset, y \notin \cup x$. Yields truth.

8. $x \cap y=\emptyset,(\forall z \in x)(z \operatorname{comp} y)$. Yields "all elements of $x$ have a common element".

9. $x \cap y=\emptyset,(\forall z \in y)(z \operatorname{comp} x)$. Yields truth.

10. $x \notin \cup y, y \notin \cup x$. Yields truth.

11. $x \notin \cup y,(\forall z \in x)(z \operatorname{comp} y)$. Yields truth.

12. $x \notin \cup y,(\forall z \in y)(z \operatorname{comp} x)$. Yields $x \neq \emptyset,\{\emptyset\}$.

13. $y \notin \cup x,(\forall z \in x)(z \operatorname{comp} y)$. Yields truth.

14. $y \notin \cup x,(\forall z \in y)(z \operatorname{comp} x)$. Yields truth.

15. $(\forall z \in x)(z$ comp $y),(\forall z \in y)(z \operatorname{comp} x)$. Yields truth.

16. $x \cap y=\emptyset, x \notin \cup y, y \notin \cup x$. Yields truth.

17. $x \cap y=\emptyset, x \notin \cup y,(\forall z \in x)(z$ comp $y)$. Yields "all elements of $x$ have a common element".

18. $x \cap y=\emptyset, x \notin \cup y,(\forall z \in y)(z \operatorname{comp} x)$. Yields $x \neq \emptyset \wedge \emptyset \notin x$.

19. $x \cap y=\emptyset, y \notin \cup x,(\forall z \in x)(z \operatorname{comp} y)$. Yields $x=\emptyset$.

20. $x \cap y=\emptyset, y \notin \cup x,(\forall z \in y)(z \operatorname{comp} x)$. Yields truth.

21. $x \cap y=\emptyset,(\forall z \in x)(z \operatorname{comp} y),(\forall z \in y)(z \operatorname{comp} x)$. Yields $x=\emptyset$.

22. $x \notin \cup y, y \notin \cup x,(\forall z \in x)(z \operatorname{comp} y)$. Yields truth.

23. $x \notin \cup y, y \notin \cup x,(\forall z \in y)(z \operatorname{comp} x)$. Yields $x \neq \emptyset,\{\emptyset\},\{\{\emptyset\}\}$.

24. $x \notin \cup y,(\forall z \in x)(z \operatorname{comp} y),(\forall z \in y)(z \operatorname{comp} x)$. Yields "there is a proper subset $y$ of $x$, not lying in $x$, which is an element of every element of $x \backslash y "$.

25. $y \notin \cup x,(\forall z \in x)(z \operatorname{comp} y),(\forall z \in y)(z \operatorname{comp} x)$. Yields truth.

26. $x \cap y=\emptyset, x \notin \cup y, y \notin \cup x,(\forall z \in x)(z$ comp $y)$. Yields $x=\emptyset$.

27. $x \cap y=\emptyset, x \notin \cup y, y \notin \cup x,(\forall z \in y)(z \operatorname{comp} x)$. Yields $x \neq \emptyset, \emptyset \notin x$, $\emptyset \notin \cup x$.

28. $x \cap y=\emptyset, x \notin \cup y,(\forall z \in x)(z \operatorname{comp} y),(\forall z \in y)(z \operatorname{comp} x)$. Yields falsity.

29. $x \cap y=\emptyset, y \notin \cup x,(\forall z \in x)(z \operatorname{comp} y),(\forall z \in y)(z \operatorname{comp} x)$. Yields $x=\emptyset$.

30. $x \notin \cup y, y \notin \cup x,(\forall z \in x)(z \operatorname{comp} y),(\forall z \in y)(z \operatorname{comp} x)$. Yields falsity.

31. $x \cap y=\emptyset, x \notin \cup y, y \notin \cup x,(\forall z \in x)(z \operatorname{comp} y),(\forall z \in y)(z \operatorname{comp} x)$. Yields falsity.

7. The combined list. The combined list from Sections $3-6$, of the formulas that are yielded, is presented in the Appendix. We do not list truth and falsity, as these are understood. The list is segregated, with the B, C, D, E list consisting of the yields from Sections 3, 4, 5, 6, respectively. 
Lemma 36. Every $\exists \forall$ formula is decided in $T$, or is equivalent, in $T$, to a disjunction $\beta_{1} \vee \beta_{2} \vee \beta_{3} \vee \beta_{4}$, where $\beta_{1}$ is on the $\mathrm{B}$ list, $\beta_{2}$ is on the $\mathrm{C}$ list, $\beta_{3}$ is on the $\mathrm{D}$ list, $\beta_{4}$ is on the $\mathrm{E}$ list, and where one, two, or three of these disjuncts may be missing. Furthermore, every such disjunction is equivalent, in $T$, to a $\exists \forall$ formula.

Proof. Let $\alpha$ be $\exists \forall$. By Lemma $5, \alpha$ is equivalent to $(\exists y)(x \in y \wedge \varphi) \vee$ $(\exists y)(y \in x \wedge \varphi) \vee(\exists y)(x=y \wedge \varphi) \vee(\exists y)(x$ inc $y \wedge \varphi)$, where $\varphi$ is a conjunction of formulas from the $\mathrm{A}^{*}$ list. By the construction of the combined list, we see that $\alpha$ is equivalent to $\beta_{1} \vee \beta_{2} \vee \beta_{3} \vee \beta_{4}$, where $\beta_{1}$ is on the $\mathrm{B}$ list or is decided, $\beta_{2}$ is on the $\mathrm{C}$ list or is decided, $\beta_{3}$ is on the $\mathrm{D}$ list or is decided, and $\beta_{4}$ is on the $\mathrm{E}$ list or is decided. If any of the $\beta$ 's are decided positively, then $\alpha$ is decided. If all of the $\beta$ 's are decided, then $\alpha$ is decided. The remaining case is where some $\beta$ is not decided, and no $\beta$ is positively decided. Remove the $\beta$ 's that are negatively decided.

Every such disjunction is obviously equivalent, in $T$, to a formula appearing in Lemma 5 . Hence it is equivalent, in $T$, to a $\exists \forall$ formula.

LEMma 37. Every $\exists \forall \vee \ldots \vee \exists \forall$ formula is decided in $T$, or is equivalent, in $T$, to a disjunction of formulas from the combined list.

\section{Proof. By Lemma 36.}

It is now convenient to take the dual and work with the negations of formulas from the combined list. This combined starred list is presented in the Appendix. Again, the list is segregated into the $\mathrm{B}^{*}, \mathrm{C}^{*}, \mathrm{D}^{*}$, and $\mathrm{E}^{*}$ lists.

Lemma 38. Every $\forall \exists$ formula is decided in $T$, or is equivalent, in $T$, to a conjunction $\varphi_{1} \wedge \varphi_{2} \wedge \varphi_{3} \wedge \varphi_{4}$, where $\varphi_{1}$ is on the $\mathrm{B}^{*}$ list, $\varphi_{2}$ is on the $\mathrm{C}^{*}$ list, $\varphi_{3}$ is on the $\mathrm{D}^{*}$ list, $\varphi_{4}$ is on the $\mathrm{E}^{*}$ list, and where one, two, or three of the conjuncts may be missing. Furthermore, every such conjunction is equivalent, in $T$, to a $\forall \exists$ formula.

Proof. This is the dual of Lemma 36.

For the rest of this section, we consider the complexity class $\forall \exists \wedge \ldots \wedge \forall \exists$ rather than $\forall \exists$. We return to a discussion of $\forall \exists(\exists \forall)$ in Section 9.

For this reason, it is convenient to present the combined starred list as the $\mathrm{F}$ list without segregation, and with repetitions removed. See the Appendix for the presentation.

The realizability of a list of formulas with at most $x$ free is identified with the sentence obtained by placing $(\exists x)$ in front of the conjunction of that list of formulas. This results in a sentence. 
Lemma 39. Every $\forall \exists \wedge \ldots \wedge \forall \exists$ formula is decided in $T$, or equivalent, in $T$, to a conjunction of formulas from the $\mathrm{F}$ list. Every formula on the $\mathrm{F}$ list is equivalent, in $T$, to $a \forall \exists$ formula.

\section{Proof. By Lemma 38.}

Lemma 40. Suppose that an F sublist contains F2, F18, or F20. Then its realizability is decided in $T$. Realizability of any such $\mathrm{F}$ sublist implies, in $T$, realizability of the $\mathrm{F}$ sublist by $x=\emptyset,\{\emptyset\}$, or $\{\{\emptyset\}\}$.

Proof. Any of F2, F18, F20 imply that $x$ be among $\emptyset,\{\emptyset\}$, or $\{\{\emptyset\}\}$. Hence the realizability of any given $\mathrm{F}$ sublist containing any of F2, F18, F20 is equivalent to realizability by at least one of $\emptyset,\{\emptyset\},\{\{\emptyset\}\}$. However, realizability by any one of these three sets is decided in $T$ because the truth value of any given formula on the $\mathrm{F}$ list with $x$ taken to be any one of these three sets is decided in $T$.

Lemma 41. Suppose that for every conjunction of formulas from the $\mathrm{F}$ list with F2, F18, F20 removed, realizability is decided in $T$. Then every $\exists(\forall \exists \wedge \ldots \wedge \forall \exists)$ sentence is decided in $T$.

Proof. Immediate from Lemmas 39 and 40.

This means that we can remove F2, F18, F20 from consideration. We call the result of this removal the $\mathrm{F}^{\prime}$ list, and it appears in the Appendix.

8. Realizable $\mathbf{F}^{\prime}$ sublists. $\exists(\forall \exists \wedge \ldots \wedge \forall \exists)$ sentences. We will show the following in $T$ :

1. The $\mathrm{F}^{\prime}$ list with $\mathrm{F} 19$ removed is realizable.

2. The $\mathrm{F}^{\prime}$ list with $\mathrm{F} 5, \mathrm{~F} 8$ removed is realizable.

3. The $\mathrm{F}^{\prime}$ list with $\mathrm{F} 5$ removed is not realizable.

4. The $\mathrm{F}^{\prime}$ list with $\mathrm{F} 8$ removed is not realizable.

5. F19, F5 imply $x=\emptyset$. F19, F8 imply $x=\emptyset$.

Item 5 is obvious by inspection.

Lemma 42. The following is provable in $T$. The $\mathrm{F}^{\prime}$ list is not realizable.

Proof. F1, F8, F19 are obviously incompatible.

We consider the following list of conditions on $x$ :

G LIST

G1. $\emptyset \notin x, \emptyset \in \cup x, x \subseteq V(\omega)$.

G2. Every $y \in x$ is an element of an infinite subset of $x$ whose elements are epsilon incomparable and pairwise disjoint.

G3. $x$ has no epsilon maximal element.

G4. No subset of $x$ lies in $x$. 
Lemma 43. The following is provable in T. Let $x$ satisfy the conditions on the $\mathrm{G}$ list. Then $x$ realizes the formulas on the $\mathrm{F}^{\prime}$ list without $\mathrm{F} 19$.

Proof. F1 is from G1; F3 from G3; F4 from G2; F5 from G4; F6 from G3; F7 from G2; F8 from G1; F9 from G3; F10 from G3; F11 from G4; F12 from G3; F13 from G3; F14 from G1; F15 from G3; F16 from G3; F17 from G2; F22 from G1.

Finally, we verify F21. Suppose the statement in question is true. Let $y$ be such a proper subset of $x$. If $y$ is infinite then it cannot be an element of any element of $x \backslash y$, since all elements of $x$ are finite. Hence $y$ is finite. Let $u$ be an infinite subset of $x$ whose elements are pairwise disjoint. Let $v, w \in u$ be distinct and not lie in $y$. Since $v, w$ are disjoint elements of $x \backslash y$, $y$ cannot lie in every element of $x \backslash y$.

The Fibonacci set sequence is defined as follows: $x_{0}=\emptyset, x_{1}=\emptyset, x_{n+2}=$ $\left\{x_{n}, x_{n+1}\right\}$.

We write $\mathrm{FIB}_{1}$ for the set of all odd numbered terms of the Fibonacci set sequence, and $\mathrm{FIB}_{2}$ for the set of all positive even number terms of the Fibonacci set sequence, i.e., $\mathrm{FIB}_{1}=\left\{x_{1}, x_{3}, \ldots\right\}$ and $\mathrm{FIB}_{2}=\left\{x_{2}, x_{4}, \ldots\right\}$.

LEMma 44. The following is provable in $\mathrm{T}$. $\mathrm{FIB}_{2}$ satisfies all conditions on the $\mathrm{G}$ list.

Proof. In the Fibonacci set sequence, by induction, the $x_{n}, n \geq 1$, are distinct elements of $V(\omega)$.

G1 is obvious. Note that for all even $n \geq 2, x_{n} \in x_{n+2}$. This verifies G3 Also for all even $n \geq 2, x_{n} \cap x_{n+4}=\emptyset, x_{n} \notin x_{n+4} \notin x_{n}$. This establishes G2. Finally, $x_{2}$ is not a subset of $x$. Also for even $n \geq 2, x_{n}$ is not a subset of $x$ since $x_{n-1} \notin x$. This establishes G4.

Lemma 45. The following is provable in $T$. The $\mathrm{F}^{\prime}$ list without $\mathrm{F} 19$ is realizable. Furthermore, it is realized by $x=\mathrm{FIB}_{2}$.

Proof. Immediate from Lemmas 43 and 44.

We now show in $T$ that the $\mathrm{F}^{\prime}$ list with $\mathrm{F} 5, \mathrm{~F} 8$ removed is realizable. We consider the following list of conditions on $x$ :

\section{H LisT}

H1. $\emptyset \in x, \emptyset \in \cup x, x \subseteq V(\omega)$.

H2. Every $y \in x$ is an element of an infinite subset of $x$ whose elements are epsilon incomparable and pairwise disjoint.

H3. $x$ has no epsilon maximal element.

Lemma 46. The following is provable in $T$. Let $x$ satisfy the conditions on the $\mathrm{H}$ list. Then $x$ realizes the formulas on the $\mathrm{F}^{\prime}$ list without $\mathrm{F} 5, \mathrm{~F} 8$. 
Proof. F1 is from H1; F3 from H3; F4 from H2; F6 from H3; F7 from $\mathrm{H} 2$; F9 from H3; F10 from H3; F11 from H2; F12 from H2; F13 from H3; F14 from H2; F15 from H3; F16 from H3; F17 from H2; F19 from H1; F22 from H1. Finally, we verify F21 exactly as in the proof of Lemma 43.

LEMMA 47. The following is provable in $T$. $\mathrm{FIB}_{1}$ satisfies all conditions on the $\mathrm{H}$ list. The $\mathrm{F}^{\prime}$ list without $\mathrm{F} 5, \mathrm{~F} 8$ is realizable by $\mathrm{FIB}_{1}$.

Proof. Since $x_{1}=\emptyset$ and $\emptyset \in x_{3}$, we see that H1 holds. The rest of the proof follows those of Lemmas 44 and 45 .

Lemma 48. The following is provable in $T$. The $\mathrm{F}^{\prime}$ list without $\mathrm{F} 5$ is not realizable. The $\mathrm{F}^{\prime}$ list without $\mathrm{F} 8$ is not realizable.

Proof. The $\mathrm{F}^{\prime}$ list without F5, F8 implies $\emptyset \in x$, using F1 and F19. Hence the $\mathrm{F}^{\prime}$ list without $\mathrm{F} 8$ is not realizable, using F5 $(\emptyset$ is a subset of $x$ lying in $x$ ), and the $\mathrm{F}^{\prime}$ list without $\mathrm{F} 5$ is not realizable, using $\mathrm{F} 8$.

This completes the verification of items $1-5$ at the beginning of this section.

Lemma 49. The following is provable in $T$. An $\mathrm{F}^{\prime}$ sublist is realizable if and only if ((it does not contain $\mathrm{F} 19)$ or (it does not contain $\mathrm{F} 5$ and it does not contain $\mathrm{F} 8$ ) or (it is realizable by $x=\emptyset)$ ).

Proof. We use items $1-5$. Suppose the $\mathrm{F}^{\prime}$ sublist $\alpha$ is realizable. If F19 is not in $\alpha$ then we are done. We assume F19 is in $\alpha$. If F5, F8 are both not in $\alpha$, then we are done. We assume F5 in $\alpha$ or F8 in $\alpha$. By 5 , we see that realizability of $\alpha$ implies the realizability of $\alpha$ by $x=\emptyset$. This completes the forward direction.

Conversely, suppose this disjunction holds. If $\alpha$ does not contain F19 then $\alpha$ is realizable by $\mathrm{FIB}_{2}$. If $\alpha$ does not contain either of $\mathrm{F} 5, \mathrm{~F} 8$, then $\alpha$ is realizable by $\mathrm{FIB}_{1}$.

Lemma 50. The realizability of any given $\mathrm{F}^{\prime}$ sublist is equivalent, in $T$, to its realizability by $x=\emptyset, \mathrm{FIB}_{1}$, or $\mathrm{FIB}_{2}$. The realizability of any given $F$ sublist is equivalent, in $T$, to its realizability by $x=\emptyset,\{\emptyset\},\{\{\emptyset\}\}, \mathrm{FIB}_{1}$, or $\mathrm{FIB}_{2}$.

Proof. The first claim is by Lemmas 45, 47, and 49. The second claim follows from the first claim and Lemma 40.

Theorem 51. Every $\exists(\forall \exists \wedge \ldots \wedge \forall \exists)$ sentence is decided in $T$. The realizability of any given $\forall \exists \wedge \ldots \wedge \forall \exists$ formula is equivalent, in $T$, to its realizability by $x=\emptyset,\{\emptyset\},\{\{\emptyset\}\}, \mathrm{FIB}_{1}$, or $\mathrm{FIB}_{2}$.

Proof. By Lemmas 39, 40, and 50. 


\section{9. $\exists \exists \forall$ sentences}

Lemma 52. Every $\exists \forall$ formula is either decided in $T$ or is equivalent, in $T$, to a disjunction of formulas on the combined list presented in the Appendix. Every $\exists \exists \forall$ sentence is either decided in $T$, or is equivalent, in $T$, to a disjunction of sentences of the form $(\exists x)(\varphi)$, where $\varphi$ is a formula on the combined list.

Proof. The first claim is by Lemma 36 . The second claim follows immediately.

LEMMA 53. Every sentence of the form $(\exists x)(\varphi)$, where $\varphi$ is a formula on the combined list, is decided in $T$. The following is provable in $T$. The realizability of any formula on the combined list is equivalent, in $T$, to its realizability by $x=\emptyset,\{\emptyset\},\{\{\emptyset\}\}$, or $\{\{\{\emptyset\}\}\}$.

Proof. We examine each entry on the combined list and give a realizing value for $x$.

B1. $x=\emptyset . \emptyset$.

C1. $x \neq \emptyset .\{\emptyset\}$.

C2. $x$ has an epsilon maximal element. $\{\emptyset\}$.

C3. Some element of $x$ is comparable with all elements of $x$. $\{\emptyset\}$.

C4. Some subset of $x$ lies in $x .\{\emptyset\}$.

C5. Some epsilon minimal element of $x$ is an epsilon maximal element of $x .\{\emptyset\}$.

C6. Some element of $x$ lies in all other elements of $x$. $\{\emptyset\}$.

C7. $\emptyset \in x .\{\emptyset\}$.

C8. $x$ has an epsilon maximum element. $\{\emptyset\}$.

C9. Some subset of $x$ is an epsilon maximal element of $x$. $\{\emptyset\}$.

C10. There is a subset of $x$ lying in $x$ which is comparable with every element of $x .\{\emptyset\}$.

C11. $x$ is a singleton. $\{\emptyset\}$.

C12. $\emptyset$ is an epsilon maximal element of $x$. $\{\emptyset\}$.

C13. $\emptyset \in x$ is an element of all other elements of $x .\{\emptyset\}$.

C14. $x$ is of the form $b \cup\{b\} .\{\emptyset\}$.

C15. $x=\{\emptyset\} .\{\emptyset\}$.

D1. $x=\emptyset$. $\emptyset$.

E1. All elements of $x$ have a common element. $\emptyset$.

E2. $x \neq \emptyset,\{\emptyset\} .\{\{\emptyset\}\}$.

E3. $x \neq \emptyset \wedge \emptyset \notin x .\{\{\emptyset\}\}$.

E4. $x=\emptyset$. $\emptyset$.

E5. $x \neq \emptyset,\{\emptyset\},\{\{\emptyset\}\} .\{\{\{\emptyset\}\}\}$. 
E6. There is a proper subset $y$ of $x$, not lying in $x$, which is an element of every element of $x \backslash y$. $\{\{\emptyset\}\}$.

E7. $x \neq \emptyset, \emptyset \notin x, \emptyset \notin \cup x . \quad\{\{\{\emptyset\}\}\}$.

THEOREM 54. Every $\exists \exists \forall$ sentence is decided in $T$. The following is provable in $T$. The realizability of any given $\exists \forall$ formula is equivalent, in $T$, to its realizability by $x=\emptyset,\{\emptyset\},\{\{\emptyset\}\}$, or $\{\{\{\emptyset\}\}\}$.

Proof. By Lemmas 52 and 53.

We conjecture that every $\exists(\exists \forall \wedge \ldots \wedge \exists \forall)$ sentence is decided in $T$.

10. $\exists \exists \wedge \ldots \wedge \exists \exists$ formulas, $\exists(\exists \exists \wedge \ldots \wedge \exists \exists)$ sentences, $\exists \forall \forall$ sentences. We use Lemma 3 and the A list.

Lemma 55. Every $\exists \exists$ formula is equivalent, in $T$, to a disjunction of formulas of the form

$$
\begin{aligned}
& (\exists y)(x \in y \wedge \varphi) \\
& (\exists y)(y \in x \wedge \varphi) \\
& (\exists y)(x=y \wedge \varphi) \\
& (\exists y)(x \text { inc } y \wedge \varphi)
\end{aligned}
$$

where $\varphi$ is on the A list.

Proof. Any $\exists$ formula is a disjunction of the formulas treated in Lemma 3 . So the $\exists \exists$ formulas are of the form $(\exists y)(\psi)$, where $\psi$ is a disjunction of formulas of the four forms appearing in Lemma 3.

LEMMA 56. Every formula of the form $(\exists y)(x \in y \wedge \varphi)$, where $\varphi$ is on the A list, is decided in $T$ or is equivalent, in $T$, to $x \neq \emptyset$.

Proof. If $\varphi$ is among $x=x, x=y, x \neq y, x \in y, y \in x, y \in \cup x$, $x$ inc $y$, then $(\exists y)(x \in y \wedge \varphi)$ is obviously decided. $(\exists y)(x \in y \wedge x \cap y \neq \emptyset)$ is equivalent to $x \neq \emptyset$, by setting $y=x \cup\{x\}$. ( $\exists y)(x \in y \wedge x \in \cup y)$ holds by setting $y=\{x,\{x\}\}$. $(\exists y)(x \in y \wedge(\exists z \in x)(z$ inc $y))$ is equivalent to $x$ $\neq \emptyset$ by setting $y=\{x,\{u\}\}$, where $u \in x .(\exists y)(x \in y \wedge(\exists z \in y)(z$ inc $x))$ holds by setting $y=\{x,\{\{x\}\}\}$.

LEMma 57. Every formula of the form $(\exists y)(y \in x \wedge \varphi)$, where $\varphi$ is on the A list, is decided in $T$ or is equivalent, in $T$, to one of the following:

$$
x \neq \emptyset
$$

"some element of $x$ lies in $\cup x$ "

"there exist two distinct epsilon incomparable elements of $x$ "

"x is not transitive".

Proof. If $\varphi$ is among $x=y, x \in \cup y, x$ inc $y$, then $(\exists y)(y \in x \wedge \varphi)$ is obviously decided. $(\exists y)(y \in x \wedge x=x),(\exists y)(y \in x \wedge x \neq y)$, and $(\exists y)(y \in x \wedge$ $y \in x)$ are obviously equivalent to $x \neq \emptyset$. $(\exists y)(y \in x \wedge x \cap y \neq \emptyset)$ is equivalent 
to $x \neq \emptyset$ by taking $y$ to be an epsilon minimal element of $x$. $(\exists y)(y \in x \wedge$ $y \in \cup x)$ is equivalent to "some element of $x$ lies in $\cup x$ ". $(\exists y)(y \in x \wedge$ $(\exists z \in x)(z$ inc $y))$ is equivalent to "there exist two epsilon incomparable elements of $x " .(\exists y)(y \in x \wedge(\exists z \in y)(z$ inc $x))$ is equivalent to $(\exists y)(y \in x \wedge$ $(\exists z \in y)(z \notin x))$ which is equivalent to " $x$ is not transitive".

LEMma 58. Every formula of the form $(\exists y)(x=y \wedge \varphi)$, where $\varphi$ is on the $\mathrm{A}$ list, is decided in $T$ or is equivalent, in $T$, to $x \neq \emptyset$.

Proof. $(\exists y)(x=y \wedge \varphi)$ is equivalent to $\varphi[y / x]$. By inspection, the $\varphi[y / x]$, where $\varphi$ is on the A list, is either decided or equivalent to $x \neq \emptyset$.

Lemma 59. Every formula of the form $(\exists y)(x$ inc $y \wedge \varphi)$, where $\varphi$ is on the A list, is decided in $T$ or is equivalent, in $T$, to " $x$ is not transitive".

Proof. If $\varphi$ is among $x=x, x=y, x \neq y, x \in y, y \in x, x \in \cup y, x$ inc $y$, then $(\exists y)(x$ inc $y \wedge \varphi)$ is decided. $(\exists y)(x$ inc $y \wedge x \cap y \neq \emptyset)$ holds by setting $y=\{\{x\}\} .(\exists y)(x$ inc $y \wedge y \in \cup x)$ is equivalent to $(\exists y)(y \in \cup x \wedge y \notin x)$ which is equivalent to " $x$ is not transitive". $(\exists y)(x$ inc $y \wedge(\exists z \in x)(z$ inc $y))$ is equivalent to $x \neq \emptyset$ by setting $y=\{\{x\}\}$. Finally, $(\exists y)(x$ inc $y \wedge$ $(\exists z \in y)(z$ inc $x))$ holds by setting $y=\{\{\{x\}\}\}$.

Here is the combined list obtained from the four lemmas.

\section{K LIST}

K1. $x \neq \emptyset$.

K2. Some element of $x$ lies in $\cup x$.

K3. There exist two distinct epsilon incomparable elements of $x$.

K4. $x$ is not transitive.

Lemma 60. Every $\exists \exists$ formula is decided in $T$ or is equivalent, in $T$, to a disjunction of formulas on the $\mathrm{K}$ list.

Proof. By Lemmas 55-59.

Lemma 61. Every $\exists \exists \wedge \ldots \wedge \exists \exists$ formula is decided in $T$ or is equivalent, in $T$, to a disjunction of conjunctions from the $\mathrm{K}$ list. Every $\exists(\exists \exists \wedge \ldots \wedge \exists \exists)$ sentence is decided in $T$ or equivalent, in $T$, to a disjunction of sentences of the form $(\exists x)(\varphi)$, where $\varphi$ is a conjunction of formulas on the $\mathrm{K}$ list.

Proof. By Lemma 60, every $\exists \exists \wedge \ldots \wedge \exists \exists$ formula is decided or equivalent to a conjunction of disjunctions of formulas on the $\mathrm{K}$ list. Therefore it is decided or equivalent to a disjunction of conjunctions of formulas on the $\mathrm{K}$ list. When we place the quantifier $(\exists x)$ in front, we can take the disjunctions out.

Lemma 62. The following is provable in $T$. The entire $\mathrm{K}$ list is realized by $x=\mathrm{FIB}_{1}$. 
Proof. By inspection.

Theorem 63. Every $\exists(\exists \exists \wedge \ldots \wedge \exists \exists)$ sentence is decided in $T$. The realizability of any given $\exists \exists \wedge \ldots \wedge \exists \exists$ formula is equivalent, in $T$, to its realizability by $x=\mathrm{FIB}_{1}$.

Proof. By Lemmas 61 and 62. By Lemma 62, every one of the sentences used in Lemma 61 of the form $(\exists x)(\varphi)$, where $\varphi$ is a conjunction of formulas on the A list, is realized by $x=\mathrm{FIB}_{1}$.

We now come to $\exists \forall \forall$. We list the negations of the formulas on the $\mathrm{K}$ list.

$\mathrm{K}^{*}$ LIST

$\mathrm{K} 1^{*} \cdot x=\emptyset$.

$\mathrm{K} 2^{*}$. No element of $x$ lies in $\cup x$.

K3*. Any two distinct elements of $x$ are epsilon comparable.

$\mathrm{K} 4^{*} . x$ is transitive.

Lemma 64. Every $\forall \forall$ formula is decided in $T$ or is equivalent, in $T$, to a conjunction of formulas on the $\mathrm{K}^{*}$ list.

Proof. By Lemma 60 using duality.

THEOREM 65. Every $\exists \forall \forall$ sentence is decided in $T$. The following is provable in $T$. The realizability of any given $\forall \forall$ formula is equivalent, in $T$, to its realizability by $x=\emptyset$.

Proof. Obviously the entire $\mathrm{K}^{*}$ list is realized by $x=\emptyset$.

\section{Two, three quantifiers. Additional results and remarks}

THEOREM 66. Every 3-quantifier sentence is decided in T. There are six sets such that every realizable 2-quantifier formula is realized by one of them. The realizability of any given 2-quantifier formula is equivalent, in $T$, to its realizability by $x=\emptyset,\{\emptyset\},\{\{\emptyset\}\},\{\{\{\emptyset\}\}\}, \mathrm{FIB}_{1}$, or $\mathrm{FIB}_{2}$.

Proof. In predicate calculus, every formula is equivalent to a formula in prenex form with the same number of quantifiers, where the quantifiers in the prenex form use distinct variables. Hence in our context, the 3-quantifier sentences are logically equivalent to sentences in the eight prenex classes given by a triple of letters from $\forall, \exists$. By Theorem 51, every $\exists(\forall \exists \wedge \ldots$ $\wedge \forall \exists$ ) sentence is decided in $T$, and every realizable $\forall \exists \wedge \ldots \wedge \forall \exists$ formula is realized within the presented list. By Theorem 54, every $\exists \exists \forall$ sentence is decided in $T$, and every realizable $\exists \forall$ formula is realized within the presented list. By Theorem 63, every $\exists(\exists \exists \wedge \ldots \wedge \exists \exists)$ sentence is decided in $T$, and every realizable $\exists \exists \wedge \ldots \wedge \exists \exists$ formula is realized within the presented list. By Theorem 65, every $\exists \forall \forall$ sentence is decided in $T$, and every realizable 
$\forall \forall$ formula is realized within the presented list. Thus we see that every $\exists \forall \exists, \exists \exists \forall, \exists \exists \exists$, and $\exists \forall \forall$ sentence is decided in $T$. Hence every 3-quantifier sentence is decided in $T$ (by taking negations). Also, note that every 2quantifier formula is $\forall \exists, \exists \forall, \exists \exists$, or $\forall \forall$, and each of these cases has been covered for the realizability claim.

We now use Lemma 38 to give an example of a $\forall \exists \wedge \ldots \wedge \forall \exists$ formula that is not equivalent to a $\forall \exists$ formula. We will use the combined starred list from the Appendix.

Let $\psi=" \emptyset \notin x \wedge x$ is not a singleton".

LEMma 67. $\psi$ is equivalent, in $T$, to a $\forall \exists \wedge \forall \exists$ formula. $\psi$ is not decided in $T$.

Proof. $\psi$ is equivalent to $(\forall y \in x)(\exists z \in y) \wedge(\forall y)(\exists z)(y \in x \rightarrow(z \in x$ $\wedge z \neq y)$ ). Note that $\psi$ holds of $\emptyset$ and fails of $\{\emptyset\}$.

In the following lemma, we use equivalence in the set-theoretic sense, and not in the sense of $T$.

LEMMA 68. $\psi$ is not equivalent to a conjunction $\varphi_{1} \wedge \varphi_{2} \wedge \varphi_{3} \wedge \varphi_{4}$, where $\varphi_{1}$ is from the $\mathrm{B}^{*}$ list, $\varphi_{2}$ is from the $\mathrm{C}^{*}$ list, $\varphi_{3}$ is from the $\mathrm{D}^{*}$ list, $\varphi_{4}$ is from the $\mathrm{E}^{*}$ list, and where one, two or three of these $\varphi$ may be missing.

Proof. Assume $\varphi_{1} \wedge \varphi_{2} \wedge \varphi_{3} \wedge \varphi_{4}$ is such a conjunction that is equivalent to $\psi$. Since $\psi$ holds of $\emptyset, \varphi_{1}$ and $\varphi_{3}$ must be missing. So we are left with a conjunction of a formula from the $\mathrm{C}^{*}$ list and a formula from the $\mathrm{E}^{*}$ list, where perhaps one of these is also missing.

We can eliminate many formulas on the $\mathrm{C}^{*}$ and $\mathrm{E}^{*}$ lists on the grounds that they are not implied by $\psi$. That is, the formula from the list fails at a set at which $\psi$ holds. Here are the eliminations and the reasons.

$\mathrm{C} 1^{*} . x=\emptyset . \psi(\{\{\emptyset\},\{\{\emptyset\}\}\})$.

C2* ${ }^{*} x$ has no epsilon maximal element. $\psi(\{\{\emptyset\},\{\{\emptyset\}\}\})$.

C3* . No element of $x$ is comparable with all elements of $x . \psi(\{\{\emptyset\}$, $\{\{\emptyset\}\}\})$.

C4*. No subset of $x$ lies in $x . \psi(\{\{\emptyset\},\{\{\emptyset\}\}\})$.

C5*. No epsilon minimal element of $x$ is an epsilon maximal element of x. $\psi(\{\{\emptyset\},\{\{\{\emptyset\}\}\}\})$.

C6* . No element of $x$ lies in all other elements of $x . \psi(\{\{\emptyset\},\{\{\emptyset\}\}\})$.

$\mathrm{C} 7^{*} . \emptyset \notin x$.

C8* $x$ does not have an epsilon maximum element. $\psi(\{\{\emptyset\},\{\{\emptyset\}\}\})$.

C9*. No subset of $x$ is an epsilon maximal element of $x . \psi(\{\{\emptyset\},\{\{\emptyset\}\}\})$.

C10*. There is no subset of $x$ lying in $x$ which is comparable with every element of $x . \psi(\{\{\emptyset\},\{\{\emptyset\}\}\})$.

C11* $x$ is not a singleton. 
$\mathrm{C} 12^{*} . \emptyset$ is not an epsilon maximal element of $x$.

$\mathrm{C} 13^{*}$. $\emptyset \notin x$ or $\emptyset$ is not an element of all other elements of $x$.

$\mathrm{C} 14^{*} . x$ is not of the form $b \cup\{b\}$.

$\mathrm{C} 15^{*} . x \neq\{\emptyset\}$.

E1*. There is no set that lies in all elements of $x . \psi(\{\{\emptyset\},\{\emptyset,\{\emptyset\}\}\})$.

$\mathrm{E} 2^{*} . x=\emptyset$ or $x=\{\emptyset\} . \psi(\{\{\emptyset\},\{\{\emptyset\}\}\})$.

$\mathrm{E} 3^{*} . x=\emptyset$ or $\emptyset \in x . \psi(\{\{\emptyset\},\{\{\emptyset\}\}\})$.

$\mathrm{E} 4^{*} . x \neq \emptyset . \psi(\emptyset)$.

$\mathrm{E} 5^{*} . x=\emptyset,\{\emptyset\}$, or $\{\{\emptyset\}\} . \psi(\{\{\emptyset\},\{\{\emptyset\}\}\})$.

E6* ${ }^{*}$. The following is false. There is a proper subset $y$ of $x$, not lying in $x$, which is an element of every element of $x \backslash y . \psi(\{\{\emptyset\},\{\emptyset,\{\emptyset\}\}\})$. Set $y=\emptyset$.

$\mathrm{E}^{*} . x=\emptyset$ or $\emptyset \in x$ or $\emptyset \in \cup x . \psi(\{\{\{\emptyset\}\},\{\{\{\emptyset\}\}\}\})$.

We are left with the following much smaller list:

$\mathrm{C} 7^{*} . \emptyset \notin x$.

$\mathrm{C} 11^{*} . x$ is not a singleton.

$\mathrm{C} 12^{*}$. $\emptyset$ is not an epsilon maximal element of $x$.

$\mathrm{C} 13^{*}$. $\emptyset \notin x$ or $\emptyset$ is not an element of all other elements of $x$.

$\mathrm{C} 14^{*} . x$ is not of the form $b \cup\{b\}$.

$\mathrm{C} 15^{*} . x \neq\{\emptyset\}$.

In particular, we have eliminated all formulas in the $\mathrm{E}^{*}$ list. But now we discard these remaining formulas on the grounds that they do not imply $\psi$ :

$\mathrm{C}^{*} . \emptyset \notin x . \neg \psi(\{\{\emptyset\}\})$.

$\mathrm{C} 11^{*} . x$ is not a singleton. $\neg \psi(\{\emptyset,\{\emptyset\}\})$.

$\mathrm{C} 12^{*}$. $\emptyset$ is not an epsilon maximal element of $x$. $\neg \psi(\{\{\emptyset\}\})$.

$\mathrm{C} 13^{*}$. $\emptyset \notin x$ or $\emptyset$ is not an element of all other elements of $x . \neg \psi(\{\{\emptyset\}\})$.

$\mathrm{C} 14^{*} . x$ is not of the form $b \cup\{b\} . \neg \psi(\{\emptyset,\{\{\emptyset\}\}\})$.

$\mathrm{C} 15^{*} . x \neq\{\emptyset\} . \neg \psi(\{\{\emptyset\}\})$.

In the second claim of the following theorem, we use equivalence in the set-theoretic sense, and not in the sense of $T$.

THEOREM 69. " $\emptyset \notin x \wedge x$ is not a singleton" is equivalent, in $T$, to a $\forall \exists \wedge \forall \exists$ formula. However, it is not equivalent to a $\forall \exists$ formula.

Proof. By Lemmas 67, 68, and 38.

As remarked in Section 1, we have discovered an example of a 5-quantifier sentence which is not decided in ZFC and is, in fact, equivalent to "there exists a subtle cardinal" over ZFC. In fact, the example is in class $\exists \forall \exists \exists \forall$. See [1], [2]. 
$[4$, p. 3] conjectures that all 7-quantifier sentences are provable or refutable in $\mathrm{ZF}$. The 5-quantifier result obviously gives a very strong refutation of this conjecture, even for $\mathrm{ZFC}+V=L$.

Furthermore, [4] remarks that the axiom of choice has eight quantifiers, as presented in the footnote on p. 3 :

$$
\begin{aligned}
\left(\forall x_{1}\right)\left(( \forall x _ { 2 } ) ( \forall x _ { 3 } ) \left(x_{2} \in x_{1} \wedge x_{3} \in x_{1}\right.\right. & \left.\rightarrow\left(\forall x_{4}\right)\left(x_{4} \notin x_{2} \vee x_{4} \notin x_{3}\right)\right) \\
\rightarrow\left(\exists x_{5}\right)\left(\forall x_{6}\right) & \left(x _ { 6 } \in x _ { 1 } \rightarrow ( \exists x _ { 7 } ) \left(x_{7} \in x_{6} \wedge x_{7} \in x_{5}\right.\right. \\
& \left.\left.\left.\wedge\left(\forall x_{3}\right)\left(x_{8} \in x_{6} \wedge x_{8} \in x_{5} \rightarrow x_{3}=x_{7}\right)\right)\right)\right) .
\end{aligned}
$$

The last two $x_{3}$ 's are typographical errors, so this should read:

$$
\begin{aligned}
\left(\forall x_{1}\right)\left(( \forall x _ { 2 } ) ( \forall x _ { 3 } ) \left(x_{2} \in x_{1} \wedge x_{3} \in x_{1}\right.\right. & \left.\rightarrow\left(\forall x_{4}\right)\left(x_{4} \notin x_{2} \vee x_{4} \notin x_{3}\right)\right) \\
\rightarrow\left(\exists x_{5}\right)\left(\forall x_{6}\right) & \left(x _ { 6 } \in x _ { 1 } \rightarrow ( \exists x _ { 7 } ) \left(x_{7} \in x_{6} \wedge x_{7} \in x_{5}\right.\right. \\
& \left.\left.\left.\wedge\left(\forall x_{8}\right)\left(x_{8} \in x_{6} \wedge x_{8} \in x_{5} \rightarrow x_{8}=x_{7}\right)\right)\right)\right) .
\end{aligned}
$$

In fact, this standard version of the axiom of choice can be put into 7-quantifier form by the following quantifier manipulations:

$$
\begin{aligned}
& \forall(\forall \forall \forall \rightarrow \exists \forall \exists \forall) \\
& \forall(\exists \exists \exists \vee \exists \forall \exists \forall) \\
& \forall \exists \exists \exists \forall \exists \forall .
\end{aligned}
$$

This already refutes the conjecture made on p. 3 of [4] that all 7-quantifier sentences can be decided in ZF.

We conjecture that all 4-quantifier sentences in set theory with $\epsilon,=$ are decided in ZF, perhaps even in $T$ plus the power set axiom.

\section{Appendix}

\section{A LisT}
A1. $x=x$.
A2. $x=y$.
A3. $x \neq y$.
A4. $x \in y$.
A5. $y \in x$.
A6. $x \cap y \neq \emptyset$.
A7. $x \in \cup y$.
A8. $y \in \cup x$.
A9. $x$ inc $y$.
A10. $(\exists z \in x)(z$ inc $y)$.
A11. $(\exists z \in y)(z$ inc $x)$. 
A* List (dual to A list)

$\mathrm{A} 1^{*} \cdot x=y$

$\mathrm{A} 2^{*} . x \neq y$.

$\mathrm{A} 3^{*} . x \notin y$.

$\mathrm{A} 4^{*} . y \notin x$.

A5*. $x \cap y=\emptyset$.

$\mathrm{A} 6^{*} . x \notin \cup y$.

$\mathrm{A} 7^{*} . y \notin \cup x$.

$\mathrm{A} 8^{*} . x$ comp $y$.

A9*. $(\forall z \in x)(z \operatorname{comp} y)$.

A10*. $(\forall z \in y)(z \operatorname{comp} x)$.

Combined List (segregated list of yields from Sections 3-6)

B1. $x=\emptyset$.

C1. $x \neq \emptyset$.

C2. $x$ has an epsilon maximal element.

C3. Some element of $x$ is comparable with all elements of $x$.

C4. Some subset of $x$ lies in $x$.

C5. Some epsilon minimal element of $x$ is an epsilon maximal element of $x$.

C6. Some element of $x$ lies in all other elements of $x$.

C7. $\emptyset \in x$.

C8. $x$ has an epsilon maximum element.

C9. Some subset of $x$ is an epsilon maximal element of $x$.

C10. There is a subset of $x$ lying in $x$ which is comparable with every element of $x$.

C11. $x$ is a singleton.

C12. $\emptyset$ is an epsilon maximal element of $x$.

C13. $\emptyset \in x$ is an element of all other elements of $x$.

C14. $x$ is of the form $b \cup\{b\}$.

C15. $x=\{\emptyset\}$.

D1. $x=\emptyset$.

E1. All elements of $x$ have a common element.

E2. $x \neq \emptyset,\{\emptyset\}$.

E3. $x \neq \emptyset \wedge \emptyset \notin x$.

E4. $x=\emptyset$.

E5. $x \neq \emptyset,\{\emptyset\},\{\{\emptyset\}\}$.

E6. There is a proper subset $y$ of $x$, not lying in $x$, which is an element of every element of $x \backslash y$.

E7. $x \neq \emptyset, \emptyset \notin x, \emptyset \notin \cup x$.

In $\mathrm{C} 3, \mathrm{C} 10$, we mean comparable in the sense of comp. 
Combined Starred List (dual to combined list)

$\mathrm{B} 1^{*} . x \neq \emptyset$.

$\mathrm{C} 1^{*} \cdot x=\emptyset$.

$\mathrm{C} 2^{*} . x$ has no epsilon maximal element.

C3* . No element of $x$ is comparable with all elements of $x$.

$\mathrm{C} 4^{*}$. No subset of $x$ lies in $x$.

C5* No epsilon minimal element of $x$ is an epsilon maximal element of $x$.

C6* ${ }^{*}$ No element of $x$ lies in all other elements of $x$.

$\mathrm{C} 7^{*} . \emptyset \notin x$.

C8* $x$ has no epsilon maximum element.

C9* No subset of $x$ is an epsilon maximal element of $x$.

C10*. There is no subset of $x$ lying in $x$ which is comparable with every element of $x$.

C11*. $x$ is not a singleton.

$\mathrm{C} 12^{*} . \emptyset$ is not an epsilon maximal element of $x$.

$\mathrm{C} 13^{*} . \emptyset \notin x$ or $\emptyset$ is not an element of all other elements of $x$.

C14* $x$ is not of the form $b \cup\{b\}$.

$\mathrm{C} 15^{*} . x \neq\{\emptyset\}$.

$\mathrm{D} 1^{*} . x \neq \emptyset$.

E1*. There is no set that lies in all elements of $x$.

$\mathrm{E} 2^{*} . x=\emptyset \vee x=\{\emptyset\}$.

$\mathrm{E}^{*} . x=\emptyset \vee \emptyset \in x$.

$\mathrm{E} 4^{*} . x \neq \emptyset$.

$\mathrm{E}^{*} . x=\emptyset \vee x=\{\emptyset\} \vee x=\{\{\emptyset\}\}$.

$\mathrm{E} 6{ }^{*}$. The following is false. There is a proper subset $y$ of $x$, not lying in $x$, which is an element of every element of $x \backslash y$.

$\mathrm{E} 7^{*} . x=\emptyset \vee \emptyset \in x \vee \emptyset \in \cup x$.

In $\mathrm{C}^{*}, \mathrm{C} 10^{*}$, we mean comparable in the sense of comp.

F LIST (unsegregated combined starred list)

F1. $x \neq \emptyset$.

F2. $x=\emptyset$.

F3. $x$ has no epsilon maximal element.

F4. No element of $x$ is comparable with all elements of $x$.

F5. No subset of $x$ lies in $x$.

F6. No epsilon minimal element of $x$ is an epsilon maximal element of $x$.

F7. No element of $x$ lies in all other elements of $x$. 
F8. $\emptyset \notin x$.

F9. $x$ has no epsilon maximum element.

F10. No subset of $x$ is an epsilon maximal element of $x$.

F11. There is no subset of $x$ lying in $x$ which is comparable with every element of $x$.

F12. $x$ is not a singleton.

F13. $\emptyset$ is not an epsilon maximal element of $x$.

F14. $\emptyset \notin x$ or $\emptyset$ is not an element of all other elements of $x$.

F15. $x$ is not of the form $b \cup\{b\}$.

F16. $x \neq\{\emptyset\}$.

F17. There is no set that lies in all elements of $x$.

F18. $x=\emptyset \vee x=\{\emptyset\}$.

F19. $x=\emptyset \vee \emptyset \in x$.

F20. $x=\emptyset \vee x=\{\emptyset\} \vee x=\{\{\emptyset\}\}$.

F21. The following is false. There is a proper subset $y$ of $x$, not lying in $x$, which is an element of every element of $x \backslash y$.

F22. $x=\emptyset \vee \emptyset \in x \vee \emptyset \in \cup x$.

$\mathrm{F}^{\prime}$ List (pruned F list)

F1. $x \neq \emptyset$.

F3. $x$ has no epsilon maximal element.

F4. No element of $x$ is comparable with all elements of $x$.

F5. No subset of $x$ lies in $x$.

F6. No epsilon minimal element of $x$ is an epsilon maximal element of $x$.

F7. No element of $x$ lies in all other elements of $x$.

F8. $\emptyset \notin x$.

F9. $x$ has no epsilon maximum element.

F10. No subset of $x$ is an epsilon maximal element of $x$.

F11. There is no subset of $x$ lying in $x$ which is comparable with every element of $x$.

F12. $x$ is not a singleton.

F13. $\emptyset$ is not an epsilon maximal element of $x$.

F14. $\emptyset \notin x$ or $\emptyset$ is not an element of all other elements of $x$.

F15. $x$ is not of the form $b \cup\{b\}$.

F16. $x \neq\{\emptyset\}$.

F17. There is no set that lies in all elements of $x$.

F19. $x=\emptyset \vee \emptyset \in x$.

F21. The following is false. There is a proper subset $y$ of $x$, not lying in $x$, which is an element of every element of $x \backslash y$.

F22. $x=\emptyset \vee \emptyset \in x \vee \emptyset \in \cup x$. 


\section{References}

[1] H. Friedman, Subtle cardinals and linear orderings, Ann. Pure Appl. Logic 107 (2001), 1-34.

[2] - , Primitive independence results, J. Math. Logic 3 (2003), 67-83.

[3] D. Gogol, The $\forall_{n} \exists$-completeness of Zermelo-Fraenkel set theory, Z. Math. Logik Grundlagen Math. 24 (1978), 289-290.

[4] - Sentences with three quantifiers are decidable in set theory, Fund. Math. 102 (1979), 1-8.

Department of Mathematics

Ohio State University

Columbus, OH 43210, U.S.A.

E-mail: friedman@math.ohio-state.edu

Web: www.math.ohio-state.edu/ friedman/

Received 14 July 2002;

in revised form 23 March 2003 